\title{
Centro de Referência em Obesidade do Município do Rio de Janeiro - 0 Papel do Educador Físico
}

\section{Reference Center on Obesity (RCO) in the City of Rio de Janeiro - The Role of Physical Educator}

\author{
Phillipe Augusto Ferreira Rodrigues' \\ Carlos Gabriel Bustamante ${ }^{1}$ \\ Erika Cardoso dos Reis ${ }^{1,2}$
}

\section{RESUMO}

O objetivo deste trabalho é apresentar a estrutura organizacional do Centro de Referência em Obesidade e o papel do educador físico na equipe para tratamento da obesidade. $\mathrm{O}$ Centro de Referência em Obesidade é formado por uma equipe multidisciplinar, aonde o educador físico vem a contribuir para a construção de um olhar mais amplo sobre o processo terapêutico através da atividade física. A atuação deste profissional se dá através de uma completa avaliação do paciente e questões associadas à prática do exercício físico. A intervenção é realizada por meio de conscientização, informação e práticas corporais em seu aspecto global. O educador físico no Centro de Referência em Obesidade busca desenvolver estudos e formular metodologias capazes de produzir evidências e comprovar a efetividade de estratégias de atividades físicas na prevenção da obesidade e na promoção da saúde.

\section{PALAVRAS-CHAVE}

Exercício; Atividade física; Perda de peso; Promoção da saúde; Qualidade de vida.

\begin{abstract}
The objective of this paper is to present the organizational structure of the Reference Center on Obesity and the function of the physical educator on staff to treat obesity. The Reference Center on Obesity consists of a multidisciplinary team, where the physical educator will contribute to building a broader look on the therapeutic process through physical activity. The work of this professional is through a thorough patient assessment and issues associated with physical exercise. Intervention is accomplished through awareness, information and bodily practices in its global aspect. The physical educator in Reference Center on Obesity seeks to develop studies and formulate methodologies able to produce evidence and prove the effectiveness of strategies for physical activity in prevention of obesity and bealth promotion.
\end{abstract}

\section{KEYWORDS}

Exercise; Physical Activity; Weight Loss; Health Promotion; Quality of Life. 


\section{INTRODUÇÃO}

A obesidade é integrante do grupo de Doenças Crônicas não transmissíveis (DCNT), sendo sua causa de origem multifatorial. No Brasil os índices de obesidade (IMC $\geq 30 \mathrm{~kg} / \mathrm{m}^{2}$ ) já alcançam $15,8 \%$ da população ${ }^{1}$. A obesidade está associada à comorbidades como hipertensão arterial sistêmica, diabetes mellitus tipo II, dislipidemia, hipotireoidismo entre outras implicações como o aumento do risco cirúrgico e anestésico. Estas características estão mais acentuadas em indivíduos com índice de massa corporal (IMC) acima de 40m/ $\mathrm{kg}^{2}$, considerados obesos de grau III, e dificultam a adesão ao tratamento, incluindo a prática de exercícios físicos ${ }^{2-6}$.

A eficácia do tratamento clínico ambulatorial realizado em caráter multidisciplinar encontra respaldo na literatura e vem sendo implementado em diversos países como uma forma não-cirúrgica para o tratamento da obesidade e sua prevenção $0^{7,8}$.

Amparado pelas perspectivas anteriores e permeado pelo atual quadro de aumento da obesidade a secretaria municipal de saúde do Rio de Janeiro em uma iniciativa pioneira, implementou em julho de 2011 o Centro de Referência em Obesidade - CRO articulado a estratégia de saúde da família, que através de uma equipe multiprofissional (médico endocrinologista, nutricionista, psicólogo, enfermeiro e educador físico) oferece tratamento clínico ambulatorial para usuários com obesidade grau III.

O objetivo deste trabalho é apresentar a estrutura organizacional do Centro de Referência em Obesidade e o papel do educador físico na equipe multiprofissional para tratamento da obesidade.

\section{CARACTERIZAÇÃO DO CRO}

O CRO é um serviço especializado no atendimento do usuário obeso e foi implantado dentro de uma Clínica da Família com a intenção de estar mais próximo das ações de saúde realizadas no território de atuação das equipes de saúde da família. As Clínicas da Família fazem parte do Programa Saúde Presente da Superintendencia de Atenção Básica do município do Rio de Janeiro e tem como objetivos trabalhar a prevenção e a promoção da saúde de seus usuários. O CRO busca desenhar um modelo de atenção à obesidade que englobe uma equipe multiprofissional e a organização dos diferentes níveis de complexidade da rede de saúde na cidade.

No CRO o objetivo de se trabalhar com uma equipe multiprofissional é a construção da multi e interdisciplinaridade para atingir um olhar mais amplo sobre o usuário. O serviço possibilita cuidados diferenciados voltados ao atendimento interdisciplinar envolvendo estratégias como consultas individuais, grupos educativos e interconsulta, baseando-se na construção compartilhada do projeto terapêutico.

De forma geral os pacientes apresentam um quadro clínico estável após o período de tratamento, e a equipe busca a manutenção do vínculo com agendamentos de consultas intervaladas e ou grupos educativos, de acordo com a singularidade de cada caso e também a possibilidade de continuidade do tratamento pelos serviços secundários de referência, como as policlínicas e ou equipes de saúde da família, de acordo com a proximidade de sua residência. 
O total de pacientes atendidos até fevereiro/2014 pelos 03 CRO é de 751 maiores de 18 anos, sendo 78\% do sexo feminino. A média de idade dos usuários adultos é de 43 anos, o que ressalta a procura pelo serviço entre usuários em idade produtiva, para ambos os sexos.

A grande maioria (mais de $80 \%$ ) dos pacientes chegam aos CRO com valores de IMC de 40,01 a $60,00 \mathrm{Kg} / \mathrm{m}^{2}$. Entretanto, pode-se observar alguns casos com IMC maior que $80,01 \mathrm{Kg} / \mathrm{m}^{2}$.

No acompanhamento de 1 a 2 anos, observou-se que $60 \%$ dos homens e $72 \%$ das mulheres conseguiram perder peso, sendo que $6 \%$ dos homens e $22 \%$ das mulheres perderam de 5 a $10 \%$ do peso. Isto demonstra que este indicador deve ser monitorado constantemente, a fim de se obter uma série histórica, guiando a assistência e mostrando tendências temporais com relação ao acompanhamento dos usuários.

Pacientes em acompanhamento regular no CRO há pelo menos um ano, com boa adesão ao tratamento, que apresentem perda de peso e manifestem desejo em realizar cirurgia bariátrica, salvo contraindicações ao procedimento, são encaminhados para o serviço de referência estadual.

Observou-se que, no período de janeiro a dezembro de 2013, dos 738 pacientes inscritos, $34,4 \%$ por algum motivo desistiram do tratamento, considerando para tal o não comparecimento ao serviço no período de 3 meses ou 3 faltas consecutivas sem justificativa, mesmo após busca ativa.

A implementação de um serviço de saúde como o CRO tem permitido avanços e identificado desafios na construção da linha de cuidado em obesidade na cidade. Os resultados iniciais da implementação do acompanhamento clínico sugerem potencialidades como a redução de dificuldade de acesso dos pacientes ao serviço de saúde, vínculo ao profissional e adesão ao tratamento para evolução clínica positiva.

Destaca-se seu caráter inovador visto que uma equipe de profissionais voltados ao acompanhamento clínico da obesidade, em uma estrutura física adequada, envolve um baixo custo relativo. Além disso, diante das experiências no cuidado da obesidade, tornam-se potenciais matriciadores para as equipes da ESF (Estratégia Saúde da Família). Este desdobramento viabiliza resolutividade na linha de cuidado em obesidade e suas comorbidades, impactando positivamente na diminuição dos agravos das DCNT na população atendida.

\section{Público Alvo}

O público alvo do CRO são usuários com obesidade grau III, atendidos por uma unidade de saúde do município do Rio de Janeiro, que através de um profissional de saúde é referenciado ao serviço.

Devido à alta demanda, os critérios de inclusão do serviço foram refinados sendo eles: Índice de Massa Corporal (IMC) superior a $40 \mathrm{~kg} / \mathrm{m}^{2}$ e diabetes ou IMC superior a $50 \mathrm{~kg} / \mathrm{m}^{2} \mathrm{com}$ ou nenhuma comorbidade associada. Optouse por estes critérios devido ao agravamento do estado de saúde conforme o aumento do IMC e sua associação com a diabetes ${ }^{9,10}$.

\section{Componentes do Programa}

Ao iniciar no serviço o paciente é recepcionado em grupo, coordenado pelo profissional de enfermagem, que explica os objetivos e o funcionamento do serviço. Em seguida tem sua primeira avaliação agendada, conforme o fluxo apresentado na figura a baixo: 


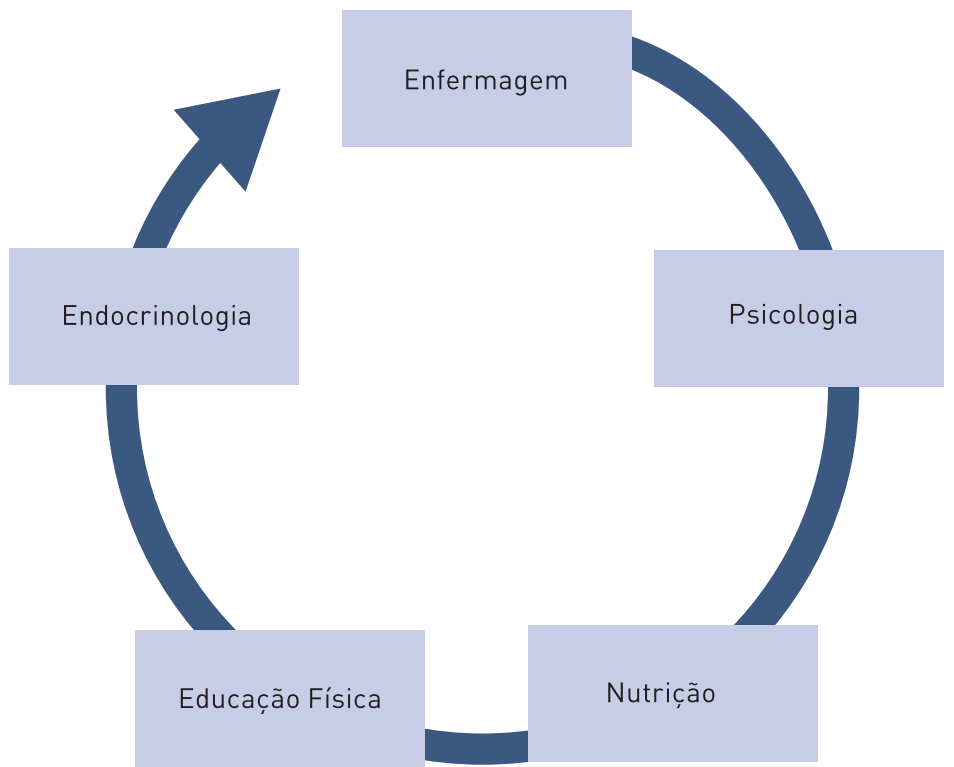

FIGURA 1 - Fluxo do Atendimento Multidisciplinar no Centro de Referência em Obesidade.

Inicialmente o paciente passa por uma avaliação clínica realizada por um profissional de enfermagem para levantamento do estado atual de saúde. Neste momento, o enfermeiro realiza uma anamnese semiestruturada com o objetivo de conhecer o histórico de saúde do usuário e de familiares, além de hábitos e comportamentos que possui, se tem outras doenças associadas e medicamentos que já faz uso. Posteriormente é realizada consulta com a psicologia para início da terapia psíquica. Em seguida são agendadas as consultas com a nutrição e educação física simultaneamente. A avaliação nutricional, realizado pelo nutricionista, visa conhecer o comportamento dos hábitos alimentares, explorar e estimular uma nova percepção sobre a necessidade de modificações nos hábitos alimentares e orientar o indivíduo a uma reeducação alimentar, valorizando a qualidade nutricional do padrão alimentar. O papel do educador físico será discutido adiante. No atendimento médico, o paciente passa por uma avaliação endocrinológica para avaliação clínica da obesidade e comorbidades associadas. Paralelo às consultas individuais, os profissionais do serviço reúnem-se semanalmente para discussão dos casos clínicos e formação de grupos terapêuticos com a participação de todas as categorias profissionais.

\section{Educador Físico no Centro de Referência em Obesidade}

Ter o educador físico inserido em um centro de referência voltado para o usuário obeso, dentro da ESF é de grande importância. O perfil dos usuários atendidos pelo serviço é caracterizado, em grande parte, por indivíduos com renda mensal de até um salário mínimo e que não têm à disposição locais para prática de atividade física orientada e gratuita. $\mathrm{O}$ educador físico, portanto, irá orientar quanto aos exercícios mais indicados caso a caso, a fim de ter certeza de que a prática do exercício irá causar benefícios à saúde. Sem acesso ao educador físico, esses indivíduos com obesidade grave poderiam optar por começar a realizar exercícios sem orientação, acreditando que isso poderá estar lhes trazendo benefícios, quando na verdade, poderão lhe estar causando malefícios se estiverem realizando de forma inadequada. 
Ao ingressar no CRO o paciente é avaliado pela equipe multidisciplinar como citado anteriormente. A avaliação física e funcional é realizada pelo educador físico sendo complementar às avaliações realizadas pelos demais profissionais. Tem como objetivo identificar possíveis impedimentos para prática de atividade física e exercícios gerando também um processo de diálogo e reflexão entre o profissional e o usuário sobre a relação do indivíduo com a prática de atividade física. Este processo se dá através de questionamentos sobre o nível de atividade física auto referido pelo indivíduo, histórico e prática atual, dificuldades e meios de vida relacionados à prática do exercício físico.

Ao final da anamnese são aplicados dois instrumentos, primeiro o questionário Short Form 36 (SF-36) para avaliação da qualidade de vida e como parâmetro complementar da avaliação da metodologia de intervenção aplicada $^{11}$. Em seguida um instrumento para avaliação da autoimagem corporal utilizando a escala de silhuetas corporais para analisar a discrepância entre a percepção do saudável, do corpo desejado e o estado atual do usuário ${ }^{12}$.

Ao final do processo avaliativo, o paciente recebe orientações gerais sobre a prática adequada de atividade física. Em seguida, o mesmo pode ser enquadrado em duas categorias, os que estão aptos e os não aptos a realizar exercício físico segundo os critérios adotados pela equipe multiprofissional. Para determinar se o usuário está apto ou não, a equipe verifica se há contraindicações importantes para o início de exercícios físicos, como cardiopatias instáveis, hipertensão arterial sistêmica descompensada, artropatias graves ou outras comorbidades que impossibilitem a realização da atividade física. Quando não está apto o paciente recebe orientações sobre o aumento das atividades diárias onde ele possa começar a fazer mais atividades inseridas no seu cotidiano e dentro do seu limite pessoal. É também convidado a participar de grupos com práticas corporais como auriculoterapia, massagem e grupo de discussão multiprofissional. O paciente passa a ser considerado apto para realização de exercício físico a qualquer momento em decisão tomada pela equipe multiprofissional.

O usuário considerado apto para exercício físico é encaminhado a grupos de exercitação física comunitária como as Academias Cariocas, Academias da Saúde, Academias da Terceira Idade entre outros grupos que ofertem a exercitação de forma gratuita e supervisionada. Quando esta não é disponível o paciente é convidado a realizar sessões de exercício físico no próprio $\mathrm{CRO}$, sendo realizados exercícios contra resistência que utilizem o próprio peso corporal, como o peso dos braços e pernas e/ou ligeira sobrecarga com halteres e elásticos para fortalecimento muscular, podendo ainda, se possível, a realização de caminhadas para melhora do sistema cardiovascular e maior perda de calorias. Esses exercícios podem ser orientados para serem realizados em casa com o objetivo de ampliar o volume de atividades realizadas pelo paciente.

É comum visualizar no usuário recém ingresso no serviço, uma falta de motivação e estímulo para iniciar um programa de exercício físico, resultando em uma baixa adesão na atividade e nesses casos, o acompanhamento psicológico deve ser reforçado. A falta de adesão é provocada também devido à baixa condição socioeconômica dos usuários que têm dificuldades de se deslocarem várias vezes ao mês para o CRO. Por este motivo, muitas atividades são orientadas para serem feitas em casa, como exercícios de baixa complexidade de coordenação e postura, alongamentos e caminhadas. Quando o usuário co- 
meça a notar melhora em sua condição física, se torna mais motivado e adere com mais facilidade ao programa de exercícios realizados no próprio CRO. Ao se tornar um usuário com adesão ao programa de exercícios, o mesmo refere melhoras no sono, no trabalho e nas atividades da vida diária, se tornando indivíduos com melhor autonomia funcional.

O exercício ainda é utilizado como forma de controle de estímulo da compulsão alimentar por consequência dos efeitos anorexígenos do exercício realizado próximo ao horário relatado de maior compulsão, além dos benefícios psicológicos promovidos pelo mesmo, como o aumento da autoestima e diminuição da depressão e ansiedade, o que vem a somar com o acompanhamento clínico.

Dentre as diretrizes da ESF estão a intersetorialidade e a multidisciplinaridade, visando à promoção, proteção e recuperação da saúde. Uma vez inserido no Centro de Referência em Obesidade o educador físico é capaz de desenvolver ações que sejam compatíveis com estas diretrizes e as metas específicas do serviço. Neste sentido, a presença do educador físico na equipe multiprofissional vem a contribuir para a construção de um olhar mais amplo sobre o paciente com obesidade grau III.

O educador físico no Centro de Referência em Obesidade realiza suas atividades baseadas nas orientações da Política Nacional de Promoção da Saú$\mathrm{de}^{13}$, mapeando e apoiando as ações de atividade física existente e inserindo ações de promoção a saúde através da atividade física onde estas ainda não estão presentes para o público obeso. Ainda dentro desta perspectiva o educador físico desenvolve estudos e formula metodologias capazes de produzir evidências e comprovar a efetividade de estratégias de atividades físicas no controle e na prevenção da obesidade.

\section{CONSIDERAÇÕES FINAIS}

O educador físico no CRO tem como objetivo proporcionar maior efetividade no tratamento ambulatorial de obesos grau III através da adoção de estratégias para mudança de hábitos sedentários sempre considerando as limitações físicas e socioeconômicas dos pacientes. Cabe ressaltar ainda, a importância de que haja estreitamento de relações com outros serviços e setores a fim de potencializar o tratamento oferecido a este público.

\section{REFERÊNCIAS}

1. Brasil. Ministério da Saúde. Estimativas sobre Frequência e Distribuição Sociodemográfica de Fatores de Risco e Proteção para Doenças Crônicas nas Capitais dos 26 Estados Brasileiros e no Distrito Federal em 2011 [online]. Brasília: Ministério da Saúde; 2012. [citado em 2014 fev 17]. Disponível em: http://bvsms.saude.gov.br/ bvs/publicacoes/vigitel_brasil_2011_fatores_risco_doencas_cronicas.pdf

2. Pinheiro ARO, Freitas SFT, Corso ACT. Uma abordagem epidemiológica da obesidade; An epidemiological approach to obesity. Rev Nutr. 2004;17:523-33.

3. Livhits M, Mercado C, Yermilov I, Janak A. Parikh JA, Dutson E, et al. Exercise following bariatric surgery: systematic review. Obesity surgery. 2010;20:657-65.

4. Gutin B. Diet vs exercise for the prevention of pediatric obesity: the role of exercise. International Journal of Obesity. 2011;35:29-32.

5. Marcon ER, Gus I, Neumann CR. Impacto de um programa mínimo de exercícios físicos supervisionados no risco cardiometabólico de pacientes com obesidade mórbida. Arq Bras Endocrinol Metab. 2011;55:331-8. 
6. Tavares TB, Nunes SM, Santos MO. Obesidade e qualidade de vida: revisão da literatura. Revista Médica De Minas Gerais. 2010;20(3):359-66.

7. Barreto SM, Pinheiro ARO, Sichieri R, Monteiro CA, Batista Filho M, Schimidt MI, et al. Análise da estratégia global para alimentação, atividade física e saúde, da Organização Mundial da Saúde. Epidemiologia e Serviços de Saúde. 2005;14(1):41-68.

8. Castelnuovo G, Manzoni GM, Cuzziol P, Cesa GL, Tuzzi C, Villa V, et al. TECNOB: study design of a randomized controlled trial of a multidisciplinary telecare intervention for obese patients with type-2 diabetes. BMC Public Health [online]; 2010;10:204 [citado 2014 fev 17]. Disponível em: http://www.ncbi.nlm.nih.gov/pmc/articles/ PMC2873580/

9. Dias JCR, Campos JADB. Aspectos epidemiológicos da obesidade e sua relação com o Diabetes mellitus. Nutrire: Rev Soc Bras Alim Nutr. 2008;33(1):103-15.

10. Sartorelli DS, Franco LJ. Tendências do diabetes mellitus no Brasil: o papel da transição nutricional. Cad Saude Publica. 2003;19(Suppl 1):S29-S36.

11. Ciconelli RM. Ferraz MB, Santos W, Meinão I, Quaresma MR. Tradução para a língua portuguesa e validação do questionário genérico de avaliação de qualidade de vida SF36 (Brasil SF-36). Rev Bras Reumatol. 1999;39(3):143-50.

12. Stunkard AJ, Sørensen T, Schulsinger F. Use of the Danish Adoption Register for the study of obesity and thinness. Res Publ Assoc Res Nerv Ment Dis. 1983;60:115-20.

13. Brasil. Ministério da Saúde. Secretaria de Vigilância em Saúde. Secretaria de Atenção à Saúde. Política Nacional de Promoção da Saúde [online]. $3^{a}$ ed. Brasília: Ministério da Saúde; 2010. [citado em 2014 fev 17]. Disponível em: http://bvsms.saude.gov.br/bvs/ publicacoes/politica_nacional_promocao_saude_3ed.pdf

ENDERECO PARA

CORRESPONDÊNCIA

PHILLIPE AUGUSTO FERREIRA

RODRIGUES

Rua João Torquato, no. 19, apto. 103

Fundos, Bonsucesso, Rio de Janeiro,

RJ, CEP: 21.032-150. (21) 9291-0090

RECEBIDO $14 / 12 / 2013$

E-mail: rodriguespafahotmail.com 